\title{
Diagnostic and prognostic micro-RNAs in ischaemic stroke due to carotid artery stenosis and in acute coronary syndrome: a four-year prospective study
}

\author{
Jacek Gacoń1, 2, Rafał Badacz², Ewa Stępieńn, Izabela Karch², Francisco J. Enguita, \\ Krzysztof Żmudka², Tadeusz Przewłocki², Anna Kabłak-Ziembicka² \\ 'Department of Invasive Cardiology, E. Szczeklik Hospital, Tarnow, Poland \\ ${ }^{2}$ Department of Interventional Cardiology, Jagiellonian University School of Medicine, John Paul II Hospital, Krakow, Poland \\ ${ }^{3}$ Department of Medical Physics, Faculty of Physics, Astronomy, and Applied Computer Science, Jagiellonian University, Krakow, Poland \\ ${ }^{4}$ Institute of Molecular Medicine, Faculty of Medicine of Lisbon, University of Lisbon, Lisbon, Portugal
}

\begin{abstract}
A b stract
Background: Circulating microRNAs (miRs) levels are potentially important diagnostic and prognostic biomarkers in acute coronary syndrome (ACS) or cerebral ischaemic events (CIE) resulting from internal carotid artery stenosis (ICAS).

Aim: This four-year prospective study aimed to compare the levels of circulating miRs in ACS vs. CIE patients, and investigate miRs potentially associated with risk of recurrent cardiovascular events.

Methods: The circulating miRs levels (miR-1-3p, miR-16-5p, miR-34a-5p, mir-122-5p, miR-124-3p, miR-133a-3p, miR-133b, miR-134-5p, miR-208b-3p, miR-375, and miR-499-5p) were compared in 43 (34 men, $57.6 \pm 10.1$ years) patients with ACS, and in 71 (47 men, $69.5 \pm 9.6$ years) with CIE due to ICAS. A four-year prospective evaluation of miRs associated with risk of cardiovascular death (CVD), myocardial infarction (MI), CIE, or all (CVD/MI/CIE) was performed.

Results: In ACS vs. CIE patients, the levels of miR-124-3p ( $p<0.001)$, miR-134-5p ( $p=0.012)$, miR-208b-3p ( $p<0.001)$, miR-34a-5p ( $p<0.001)$, and miR-499-5p ( $<<0.001)$ were higher, while levels of miR-16-5p $(p<0.001)$ and miR-122-5p $(p<0.001)$ were lower. Levels of miR-1-3p ( $p=0.195)$, miR-133a-3p $(p=0.333)$, miR-133b $(p=0.056)$, and miR-375 $(p=0.055)$ were non-statistically different. During follow-up (median 57 months, Q1-Q3: 54-60), CVD/MI/CIE occurred in 23 subjects, including eight CVDs, five non-fatal CIEs, and 10 non-fatal MIs. The multivariate Cox proportional hazard analysis (relative risk [RR]; 95\% confidence interval $[\mathrm{CI}]$ ) revealed that miR-208b-3p (1.225; 1.092-1.375), miR-34a-5p (0.963; 0.935-0.992), and miR-499-5p (0.077; 0.025-0.239) were independently associated with risk of CVD/MI/CIE, as well as risk of each event. Furthermore, miR-133b (1.009; 1.003-1.015) was associated with risk of CVD.

Conclusions: This study shows that although most investigated miRs levels differ significantly between patients with ACS and CIE, similar levels of circulating miR-1-3p, miR-133a-3p, miR-133b, and miR-375 were observed; furthermore, we identified several common miRs as possible risk factors for recurrent cardiovascular events.
\end{abstract}

Key words: circulating miRs, acute coronary syndrome, cerebral ischaemic event, prognostic miRs

Kardiol Pol 2018; 76, 2: 362-369

\section{INTRODUCTION}

Endothelial senescence is thought to play a vital role in atherosclerosis [1]. Mechanisms of senescence include endothelial dysfunction, arterial stiffness, and endothelial microvesicle release, which seem to be vectors transferring macromolecules from originated to descending cells, including a specific group of regulatory factors - microRNAs (miRs) [2]. This family of small noncoding RNAs interacts with selected targeted mRNAs and typically represses their activity [3].

\section{Address for correspondence:}

Anna Kabłak-Ziembicka, MD, PhD, Department of Interventional Cardiology, Jagiellonian University School of Medicine, John Paul II Hospital, ul. Prądnicka 80, 31-202 Kraków, Poland, e-mail: kablakziembicka@op.pl

Received: 29.05.2017 Accepted: 16.11.2017 
Atherosclerosis can be regulated by a variety of miRs having an impact on vascular inflammation, smooth muscle cell proliferation, and plaque progression [4]. Eventually these processes lead to atherothrombotic events that may manifest as acute coronary syndrome (ACS), cerebral ischaemic event (CIE) due to large vessel atherosclerotic disease, e.g. internal carotid athero-occlusive disease (ICAS) or limb ischaemia [5, 6].

Acute coronary syndrome occurs in approximately 50\% of patients without prodromal symptoms, while unfavourable clinical course following ACS is associated with myocardium remodelling due to cardiomyocyte necrosis resulting in the development of heart failure, arrhythmia, and sudden cardiac death recurrent myocardial ischaemia and atherosclerosis progression [5, 6]. In clinical practice, several imaging and laboratory prognostic biomarkers are in use to assess a risk of congestive heart failure, sudden cardiac death or recurrent ACS, including B-type natriuretic peptides, cardiac troponins, growth-differentiation factor-15, galectin-3, heart magnetic resonance imaging or echocardiographic study showing left ventricle remodelling, as well as reperfusion time [6, 7].

Cerebral ischaemic event associated with ICAS, has the most unfavourable prognosis, about $30 \%$ of affected subjects die due to CIE during the first year after the event and 30\% become permanently disabled [8]. Recurrent CIE in the same cerebral territory is observed in $10-25 \%$ of patients within the first year [8]. However, brain computed tomography displays infarcted zone ischaemia, which is usually related to the degree of neurological deficits, and further prognosis is unpredictable due to a limited number of laboratory specific biomarkers, as yet [8].

Despite great progress in the management of atherothrombotic complications (revascularisation, medications improving survival), new potential biomarkers of ACS and CIE risk are of utmost importance.

Nowadays, promising biomarker candidates include circulating miRs that are involved in the regulatory mechanisms of physiological and pathological processes, such as smooth muscle cell proliferation and differentiation, apoptosis, metabolism, as well as neoangiogenesis and inflammation [4, $9,10]$. Furthermore, the release of cytokines and miRs as a consequence of the ischaemic event in one territory may lead to destabilisation of atherosclerotic plaques in another arterial territory $[11,12]$.

The present study aimed to compare the circulating miRs levels in ACS vs. CIE patients, and prospectively investigate them as potential biomarkers associated with risk of recurrent cardiovascular events. We aimed to identify circulating miRs that may be the prognostic markers of patients' survival following ACS and CIE resulting from ICAS in respect to further cardiovascular events (new ACS, new CIE, and cardiovascular death [CVD]). Our choice for selection of particular miRs was based on database research, taking into consideration their abundance in the organs affected by the disease (heart or brain) as well as peripheral tissues (liver or pancreas) and serum stability.

\section{METHODS \\ Groups subsets}

Prospective evaluation included 114 patients with $\mathrm{CIE}$ (group I, 71 patients) or ACS (group II, 43 patients) resulting from the atherothrombotic plaque, referred to our institution between January 2011 and December 2012 for interventional management (carotid artery revascularisation or coronary angiography and subsequent coronary intervention).

The study was performed in accordance with the Declaration of Helsinki. The protocol was reviewed and approved by a Local Ethics Committee.

\section{Inclusion criteria}

In ACS subjects at least one infarct-related lesion in a coronary artery must have been identified during coronary angiography performed prior to coronary artery revascularisation with a primary percutaneous coronary angioplasty with stent implantation.

In CIE subjects, appropriate neurological symptoms, an associated ICAS exceeding $70 \%$ lumen reduction in the territory of cerebral ischaemia, and brain imaging findings relevant to the symptoms and ICAS were required.

All patients obtained medical treatment according to guidelines of the European Society of Cardiology, and the Society of Neurology.

\section{Exclusion criteria included}

In the ACS group: patients with acute heart failure or with congestive heart failure in New York Heart Association classes III and IV, as well as patients with diagnosed ACS but with normal coronary arteries were not included into the study. In the CIE group: previous CIE, lack of the consistency between ICAS site and neurological lesions on brain computed tomography or neurological deficits identified during neurologic consultation were grounds for exclusion because this might suggest aetiology of CIE other than ICAS. Also excluded were patients with normal carotid arteries, or patients referred for thrombolysis for acute stroke. Finally, patients with asymptomatic ICAS were not included into the present study.

Other general exclusion criteria included a history of cancer, systemic inflammatory conditions such as arthritis, and known or suspected bacterial or viral infections.

Every patient was subjected for a full-spectrum laboratory analysis (miRs, biochemical parameters, and selected biomarkers) before revascularisation.

\section{Acquisition of medical records}

The distribution of classic risk factors (diabetes, hyperlipidaemia, arterial hypertension, smoking current or previous, renal kidney disease, peripheral occluded arterial disease) was recorded. Definitions of the above were adopted from the scientific statements of the European Society of Cardiology (http://www.escardio.org). 


\section{Coronary angiography and revascularisation procedure}

Coronary angiography was performed from the femoral or radial approach. A loading dose of aspirin of $300 \mathrm{mg}$, followed by $75 \mathrm{mg} /$ day was given indefinitely, and a loading dose of clopidogrel of $600 \mathrm{mg}$, followed by $75 \mathrm{mg} /$ day for 12 months. During the procedure unfractionated heparin was administrated according to patient weight and activated clotting time. Glycoprotein Ilb/IIla inhibitors were used at the operator's discretion. Other medications, such as analgesics and beta-blockers were administrated as indicated. During the revascularisation procedure, the target vessel was routinely stented, and predilatation and stent choice depended on the clinical circumstances and the operator's decision.

\section{Neurological assessment}

Neurological examination was provided in all subjects with CIE on admission to our Department by a consultant neurologist. The data of CIE (ischaemic stroke or transient ischaemic attack) were obtained from a stroke unit, and sourced from available medical documentation, obtained from brain imaging either with computed tomography or magnetic resonance imaging to assess the degree of cerebral infarction, if any, and to exclude other disorders that might cause the symptoms (e.g. subdural hematoma, tumour).

Patients were referred to carotid artery stenting (CAS) or carotid endarterectomy (CEA) on the basis of a multidisciplinary working team including a consultant neurologist, vascular surgeon, endovascular specialist, cardiologist, and radiologist, taking into account the clinical presentation, accompanying comorbidities (e.g. renal dysfunction, respiratory tract disease, access site, coronary artery disease), anatomic assessment (limited surgical access, prior cervical irradiation, prior ipsilateral CEA, and contralateral carotid occlusion), data from imaging workup, and method feasibility and safety.

\section{Angiography and ICAS revascularization}

Patients were selected for CAS if they had symptomatic $>70 \%$ lumen stenosis by quantitative carotid angiography using the Coroscop system (Siemens AG, Munich, Germany) equipped with Quantcor version 4.0 quantitative analysis software. The degree of ICAS was assessed according to North American Symptomatic Carotid Endarterectomy Trial (NASCET) criteria by measuring the vessel diameter in the point of maximal stenosis compared with the plaque-free vessel diameter distally from the lesion. Angiograms were performed in at least two orthogonal projections that best displayed the lesion.

The detailed CAS technique was at the operator's discretion, provided that the 'tailored-CAS' algorithm was applied, allowing for optimal choice of neuroprotection device and stent type depending on the lesion morphology and the neurological status of the patient [13]. CEA technique was at the operator's discretion, with preference of eversion technique whenever feasible. Periprocedural medical management contained antiplatelet treatment. All patients obtained optimal medical treatment in concordance with recommendations of respective societies.

\section{Laboratory tests}

The standard blood tests included high-sensitivity C-reactive protein (hs-CRP), fibrinogen levels, and lipid profile.

\section{miRNA extraction}

Serum samples were collected on patient admission. Samples were allowed to coagulate for $30 \mathrm{~min}$, centrifuged, and sera were frozen at $-80^{\circ} \mathrm{C}$ until miRNA and selected biomarkers analysis. Extraction of miRNA was performed by means of the miRNeasy Serum/Plasma Kit (Qiagen) with the beginning lysis by Trizol LS Reagent (Invitrogen). The RNA yield and concentrations were determined by capillary electrophoresis on the Agilent Bioanalyser 2100 with the Eukaryote Total RNA Pico Chip (Agilent Technologies, Inc., Santa Clara, CA). An average $60 \pm 31.9 \mathrm{pg} / \mu \mathrm{L}$ of total RNA from $300 \mu \mathrm{L}$ of serum was recovered.

\section{Profiling of candidate miRNAs}

At the time of the study, Exiqon LNA primers were used to quantify 10 mature miRNAs using the Viia7 real-time polymerase chain reaction (PCR) system equipped with a 384-well reaction plate (Life Technologies). RNA was converted to cDNA using the Universal cDNA Synthesis Kit (Exiqon) following the manufacturer's instructions. Real-time PCR was performed in triplicate with SYBR Green master mix Universal RT (Exiqon) using standard conditions. The organ-specific candidate miRNAs were selected as follows: cardiac and skeletal muscle-related (miR-1-3p, -133a-3p, -133b, -208b-3p, $-499-5 p)$, brain-related (miR-34a-5p, -124-3p, -134-5p), liver-specific (miR-122-5p), pancreas-specific (miR-375), and miR-16-5p. The fold changes (RQ) were calculated and statistically significant variations between groups of samples filtered by the calculation of adjusted p-values using the Benjamini-Hochberg false discovery rate.

\section{Follow-up}

The incidences of CVD, myocardial infarction (MI), and recurrent $\mathrm{CIE}$, as well as composite end-point (CVD/MI/CIE), were recorded prospectively in 112 patients during a mean follow-up period of $53.8 \pm 14.9$ months, median 57 (Q1-Q3: 54-60) months. The minimum follow-up period was three months, due to CVD.

Myocardial infarction was diagnosed according to criteria of the European Society of Cardiology. Diagnosis of CIE was to be given by a neurologist to ensure reliability. CVD was defined as a fatal CIE, fatal MI, or other CVD (i.e. any sudden or unexpected death unless proven as non-cardiovascular on autopsy).

\section{Statistical analysis}

Continuous variables are presented as mean \pm one standard deviation, and categorical variables are expressed as frequen- 
Table 1. Characteristics of study groups

\begin{tabular}{|c|c|c|c|}
\hline & $\begin{array}{l}\text { Patients with acute coronary } \\
\text { syndrome }(n=43)\end{array}$ & $\begin{array}{l}\text { Patients with ischaemic stroke } \\
\qquad(\mathrm{n}=71)\end{array}$ & p \\
\hline Age [years] & $57.6 \pm 10.1$ & $69.4 \pm 9.6$ & $<0.001$ \\
\hline Male & $34(79.1 \%)$ & $47(66.2 \%)$ & 0.142 \\
\hline Smoking & $29(67.4 \%)$ & $43(60.6 \%)$ & 0.465 \\
\hline Diabetes & $20(46.5 \%)$ & $29(40.8 \%)$ & 0.558 \\
\hline Arterial hypertension & $33(76.7 \%)$ & $67(94.4 \%)$ & 0.005 \\
\hline Dyslipidaemia & $36(83.7 \%)$ & $60(82.2 \%)$ & 0.785 \\
\hline Creatinine $[\mu \mathrm{mol} / \mathrm{L}]$ & $77 \pm 15$ & $90.9 \pm 32.9$ & 0.010 \\
\hline Chronic renal failure (eGFR $<60 \mathrm{~mL} / \mathrm{min}$ ) & $6(14 \%)$ & $17(23.9 \%)$ & 0.201 \\
\hline \multicolumn{4}{|l|}{ Concomitant arterial occlusive disease: } \\
\hline Carotid stenosis $>50 \%$ & $3(7 \%)$ & $71(100 \%)$ & $<0.001$ \\
\hline Significant CAD* & $43(100 \%)$ & $37(52.1 \%)$ & $<0.001$ \\
\hline PAOD & $6(14 \%)$ & $18(25.3 \%)$ & 0.15 \\
\hline Previous PCl & $3(6.9 \%)$ & $6(8.4 \%)$ & 0.777 \\
\hline Previous CABG & $1(2.3 \%)$ & $3(4.2 \%)$ & 0.593 \\
\hline Previous MI & $3(6.9 \%)$ & $13(18.3 \%)$ & 0.091 \\
\hline Previous CIE & $1(2.3 \%)$ & $0(0 \%)$ & 0.197 \\
\hline LDL-cholesterol [mmol/L] & $3.8 \pm 1.3$ & $2.7 \pm 1.0$ & $<0.001$ \\
\hline hs-CRP [g/L] & $10.3 \pm 16$ & $4.7 \pm 5.9$ & 0.010 \\
\hline Fibrinogen [g/L] & $4.0 \pm 1.8$ & $3.9 \pm 1.2$ & 0.709 \\
\hline
\end{tabular}

Data are shown as mean and standard deviation or number (percentage). *Significant CAD or carotid artery stenosis — angiographically diagnosed at least 50\% lumen reduction; CABG — coronary artery bypass grafting; CAD — coronary artery disease; CIE — cerebral ischemic event; eGFR - estimated glomerular filtration rate; hsCRP — high-sensitivity C-reactive protein; LDL — low-density lipoprotein; MI — myocardial infarction; PAOD — peripheral artery occlusive disease; PCI — percutaneous coronary intervention

cies and percentages. Means of analysed parameters across groups were tested with analysis of variance (ANOVA) test, and frequencies were compared by the $\chi^{2}$ test for independence. The normal distribution of studied variables was determined by the Shapiro-Wilk test. Differences between mean values of miRs were verified using the Mann-Whitney $U$ test because the distribution of variables was found not to be normal.

The potential independent prognostic markers of cardiovascular events during the follow-up period were established from the 30 variables with univariate analysis (miRs, age, gender, atherosclerosis risk factors, type of ischaemic event). If there was a trend toward difference $(p<0.1)$, they were entered into a multivariate Cox proportional hazard analysis model. The results of the multivariate logistic regression analysis were expressed as hazard ratio (HR) and 95\% confidence interval $(95 \% \mathrm{Cl})$. Statistical analyses were performed with Statistica 12.0 software. Statistical significance was assumed at $p<0.05$.

\section{RESULTS}

Group I comprised 71 consecutive subjects (47 men, mean age $69.5 \pm 9.6$ years) who suffered from CIE with a significant ICAS on angiography (mean stenosis degree $83.9 \pm 15 \%$ ), and who underwent successful carotid artery revascularisation.
Group II comprised 43 consecutive patients (34 men, mean age $57.6 \pm 10.1$ years) admitted with ACS, who underwent coronary angiography and subsequent coronary intervention according to guidelines. The detailed study group characteristics are presented in Table 1. In brief, patients with CIE were older $(p<0.001)$, more often hypertensive $(p=0.005)$, and they had higher creatinine levels $(p=0.01)$, as compared to ACS subjects. There was higher levels of hs-CRP ( $p=0.01)$ and low-density lipoprotein cholesterol $(p<0.001)$ in the ACS vs. CIE groups (Table 1$)$.

In ACS patients, as compared to CIE patients, there was a statistically significant increase of circulating miR-124-3p (log $2^{-\Delta \mathrm{Ct}} 0.571 \pm 0.07$ vs. $\left.-0.638 \pm 0.06 ; p<0.001\right)$, miR-134-5p $\left(\log 2^{-\Delta \mathrm{Ct}} 0.278 \pm 0.09\right.$ vs. $\left.-0.02 \pm 0.07 ; p=0.012\right), \mathrm{miR}-208 \mathrm{~b}-$ $-3 p\left(\log 2^{-\Delta C t}-0.899 \pm 0.08\right.$ vs. $\left.-1.998 \pm 0.149 ; p<0.001\right)$, miR-34a-5p $\left(\log 2^{-\Delta \mathrm{Ct}} 0.238 \pm 0.08 v s .-0.248 \pm 0.07 ; p<0.001\right)$, and miR-499-5p (log $2^{-\Delta \mathrm{Ct}}-0.899 \pm 0.08$ vs. $-1.176 \pm 0.07$; $p<0.001$; Fig. 1). Decreased levels of miR-16-5p $\left(\log 2^{-\Delta \mathrm{Ct}}\right.$ $-0.072 \pm 0.08$ vs. $1.962 \pm 0.103 ; p<0.001)$ and miR-122$-5 \mathrm{p}\left(\log 2^{-\Delta \mathrm{Ct}}-0.076 \pm 0.08\right.$ vs. $\left.1.892 \pm 0.09 ; \mathrm{p}<0.001\right)$ were found (Fig. 1).

Prospective evaluation was available for 112 subjects. CIE and ACS patients were considered together, as one group. 


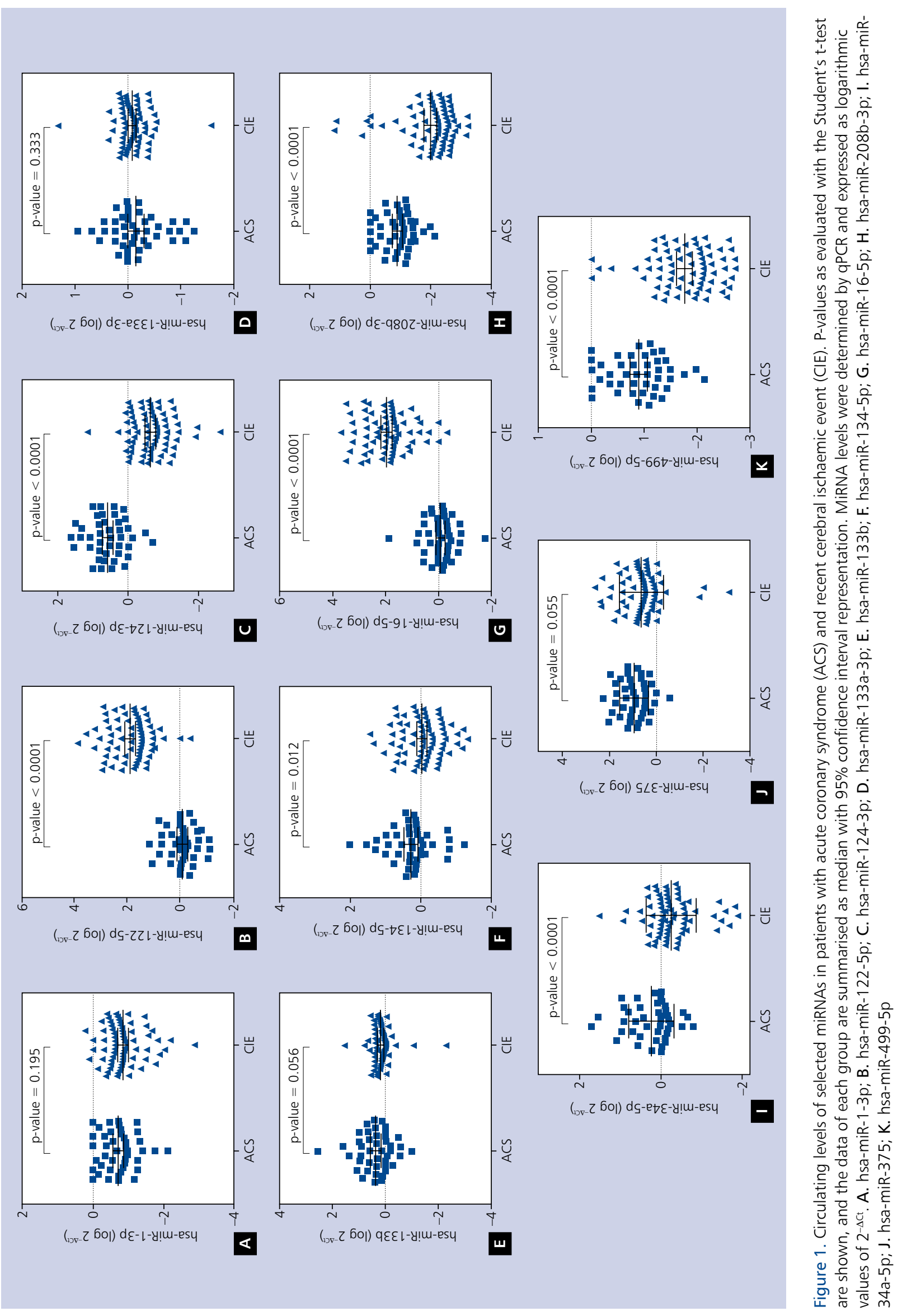


Table 2. The independent predictors of cardiovascular events identified by multivariate Cox proportional hazard analysis

\begin{tabular}{|c|c|c|c|}
\hline & HR & $95 \% \mathrm{Cl}$ & $\mathbf{p}$ \\
\hline \multicolumn{4}{|l|}{ CVD/MI/CIE } \\
\hline miR-122-5p & 1.001 & 0.999-1.001 & 0.054 \\
\hline miR-208b-5p & 1.225 & $1.092-1.375$ & 0.001 \\
\hline$m i R-34 a-5 p$ & 0.963 & $0.935-0.992$ & 0.012 \\
\hline miR-499-5p & 0.077 & $0.025-0.239$ & $<0.001$ \\
\hline \multicolumn{4}{|l|}{ CVD } \\
\hline miR-133b & 1.009 & $1.003-1.015$ & 0.004 \\
\hline miR-208b-5p & 1.133 & $1.026-1.251$ & 0.014 \\
\hline miR-34a-5p & 0.964 & $0.936-0.992$ & 0.011 \\
\hline miR-499-5p & 0.116 & $0.043-0.310$ & $<0.001$ \\
\hline \multicolumn{4}{|l|}{ CIE } \\
\hline miR-122-5p & 1.001 & $1.000-1.001$ & 0.007 \\
\hline miR-208b-5p & 1.159 & $1.043-1.288$ & 0.006 \\
\hline miR-34a-5p & 0.971 & $0.944-0.998$ & 0.037 \\
\hline miR-499-5p & 0.139 & $0.054-0.358$ & $<0.001$ \\
\hline miR-16-5p & 1.001 & $1.000-1.001$ & 0.059 \\
\hline \multicolumn{4}{|l|}{ MI } \\
\hline miR-122-5p & 1.000 & 0.999-1.001 & 0.063 \\
\hline miR-208b-5p & 1.216 & $1.087-1.359$ & 0.001 \\
\hline miR-34a-5p & 0.962 & $0.933-0.991$ & 0.010 \\
\hline miR-499-5p & 0.074 & $0.024-0.226$ & $<0.001$ \\
\hline
\end{tabular}

$\mathrm{Cl}$ - confidence interval; CIE — cerebral ischaemic event; CVD cardiovascular disease; HR — hazard ratio; $\mathrm{Ml}$ - myocardial infarction

During a four-year follow-up period, CVD/MI/CIE occurred in $23(20.5 \%)$ subjects. In the CIE group there were eight CVDs (one due to CIE and seven due to $\mathrm{MI}$ ) and five non-fatal CIEs. In the ACS group there were 10 non-fatal MIs, while no CVD or CIE were noted.

The multivariate Cox proportional hazard analysis revealed the following predictors of CVD/MI/CIE: miR-208b-3p $(H R=1.225,95 \% \mathrm{Cl} 1.092-1.375 ; p=0.001), \operatorname{miR}-34 a-5 p$ $(H R=0.963,95 \% \mathrm{Cl} 0.935-0.992 ; p=0.012)$, and miR-499$-5 p(\mathrm{HR}=0.077,95 \% \mathrm{Cl} 0.025-0.239 ; \mathrm{p}<0.001$; Table 2).

Furthermore, the levels of circulating miR-208b, miR-34a, and miR-499 occurred as independent risk factors for composite and each individual end-point (MI, CIE, and CVD; Table 2). Levels of miR-133b were identified as risk factors of future CVD (Table 2).

\section{DISCUSSION}

The major finding in the present study is that despite the same (e.g. atherothrombotic) aetiology, resulting in an ischaemic event, the levels of several circulating miRs significantly differed between subjects with ACS and CIE. To our knowledge, this is the first study comparing serum miRs levels in subjects with ACS vs. CIE. We identified significant differences in the increase of miR-124-3p, miR-134-5p, miR-34a-5p, miR-208b$-3 p$, and miR-499-5p, and in the decrease of miR-16-5p and miR-122-5p in ACS vs. CIE patients. This finding might be attributed to the different mechanism of ACS and CIE, e.g. the plaque rupture with subsequent thrombus formation is observed predominantly in ACS as compared to the embolic aetiology from frequently ulcerated soft carotid artery plaque in CIE [12]. The other potential explanation is that miRs expression depends on its release from atherosclerotic plaques, platelets, cardiomyocytes, and brain cells exposed to ischaemia $[14,15]$.

Cipollone et al. [16] found that miR-100, -127, -133a, $-133 b$, and -145 were significantly upregulated in symptomatic carotid plaques, while Raitoharju et al. [17] found that miR-21, $-34 a,-146 a,-146 b-5 p$, and -210 were the most upregulated miRNAs in atherosclerotic plaques from peripheral arteries (carotid, femoral, and abdominal aorta). In human coronary atherosclerotic plaques, at least one vascular bed, compared with a healthy arterial wall, showed different expression of miR-126, -134, -145, -146a, -198, -210, -340*, and $-92 a$ [16, 17].

Interestingly the levels of circulating miR-1-3p, miR-133a-3p, miR-133b, and miR-375 were non-significantly different between ACS and CIE groups, suggesting perhaps the same release mechanism from the plaque.

\section{miR and ACS}

Olivieri et al. [18] demonstrated a 3- to 10-fold increase in expression of miR-1, miR-133a, miR-21, and miR-423-5p and over 80-fold increase of miR-499-5p, the latter was an important diagnostic marker of non-ST elevation MI (NSTEMI) in 92 elderly subjects. Adachi et al. [19], in a miR array analysis, reported that miR-499 is almost specifically expressed in the heart, concluding that miR-499 might serve as an additional promising biomarker of MI. As shown in a mouse model, also miR-208 seems to be exclusively expressed in cardiomyocytes and consequently released during cardiomyocyte death in MI [20].

Tang et al. [21] evaluated the effect of ischaemia reperfusion on miRs in the rat heart, identifying that levels of miR-1, miR-126, and miR-208 were increased, while miR-21, miR-133, and miR-195 levels were decreased. In our previous study, we found a 3.83-fold higher miR-134 expression $(p<0.025)$ in patients with ST elevation MI-ACS as compared to patients with NSTEMI-ACS [22]. While, the main variance in miR expression was associated with patent vs. occluded target lesion in a coronary artery [23].

\section{miR and CIE}

Jickling et al. [11] showed significant down-expression of miR-122, -148a, -19a, and -320, and up-expression of miR-363 and $-487 \mathrm{~b}$ in patients with any acute ischaemic 
stroke compared to controls with vascular risk factors. In this study group, atrial fibrillation was similarly prevalent in CIE vs. controls ( $20.8 \%$ vs. $25 \%, p=0.74)$, and other potential CIE aetiologies were not examined [11]. These authors concluded that certain miRs may be differentially expressed according to the subtype of ischaemic stroke, which would require a larger cohort to examine [11]. The downregulation of miR-122 in stroke is also consistent with our current findings.

Unfortunately, data regarding the relationship between destabilisation or progression of carotid artery stenosis resulting in CIE are scarcely reported. Dolz et al. [23] showed that plaque progression in asymptomatic carotid artery stenosis was associated with high likelihood of CIE and significantly higher expression of miR-199b-3p, miR-27b-3p, miR-130a-3p, miR-221-3p, and miR-24-3p. According to previous studies, miR-146a was found to be upregulated in atherosclerotic plaques obtained from human carotid arteries while miR221, miR-100, miR-133a, or miR-145 were significantly overexpressed in unstable human carotid plaques [16, 17]. The lack of a significant difference between the levels of miR-133a observed in our study may potentially explain the common mechanism of plaque destabilisation as a process independent of plaque location.

\section{miRs and cardiovascular prognosis}

The other major finding from this study is that although miR levels differ significantly with regard to the organ exposed to ischaemia, we can identify several common miRs independently associated with future recurrent events, e.g. CVD, MI, CIE as well as composite end-point: CVD/MI/CIE. The following independent predictors of $\mathrm{CVD} / \mathrm{MI} / \mathrm{CIE}$ were identified: miR-208b $(H R=1.225,95 \% \mathrm{Cl} 1.092-1.375 ; p=0.001)$, miR-34a $(H R=0.963,95 \% \mathrm{Cl} 0.935-0.992 ; p=0.012)$, and miR-499 (HR =0.077, 95\% Cl 0.025-0.239; $p<0.001)$.

This finding might be clinically very useful because an ischaemic event in one vascular territory may trigger another ischaemic event in the same or different arterial territory. Thus, identification of miRs, presumably key players in plaque transformation from the stable into the unstable or prone to rupture, may identify new therapeutic targets.

Our present study demonstrated that the levels of serum miR-208b, miR-34a, miR-133b, and miR-499 were associated with CVD risk. In the study of 444 patients with ACS by Widera et al. [24], the admission levels of miR-133a and miR-208b were significantly associated with the risk of all-cause death at six months in univariate and age- and gender-adjusted analyses. Gidlof et al. [25], in a group of 424 patients with ACS found the independent association between miR-208b (OR = 1.79, $p<0.001)$ and miR-499-5p $(\mathrm{OR}=1.7, \mathrm{p}<0.001)$ with increased risk of mortality and heart failure within 30 days.

Furthermore, the levels of miR-208b, miR-34a, and miR499 proved to be independent risk factors of composite and particular end-points (MI, CIE, and CVD). While, the expression of miR-133b was identified as a risk factor of further CVD, and miR-16 proved to be specific for CIE incidence.

\section{Limitations of the study}

One obvious limitation is the pre-selection of miRs potentially associated with ACS or CIE, thus other miRs may also contribute to pathogenesis and prognosis in atherothrombotic events.

\section{CONCLUSIONS}

To conclude, in order to develop a target-specific therapy, it is essential to establish the exact mechanisms driving the release of specific miRs during atherosclerosis progression and remodelling. It is still not known if miRs responsible for neoangiogenesis or smooth muscle cell proliferation (miR-133a/133b) can contribute to plaque conversion from a stable to an unstable state, or if the cells exposed to ischaemia (cardiomyocytes) can release microvesicles loaded with specific miRs (miR-208 or miR-499), which remotely affect formation to increase the risk of future cardiovascular events.

Funding: The study was supported by research grant number K/ZDS/005730 to Anna Kablak-Ziembicka received from the Jagiellonian University Collegium Medicum, Krakow.

\section{Conflict of interest: none declared}

\section{References}

1. Stępień E, Kabłak-Ziembicka A, Czyż J, et al. Microparticles, not only markers but also a therapeutic target in the early stage of diabetic retinopathy and vascular aging. Expert Opin Ther Targets. 2012; 16(7): 677-688, doi: 10.1517/14728222.2012.691 471, indexed in Pubmed: 22616736.

2. Faragher RGa, McArdle A, Willows A, et al. Senescence in the aging process. F1000Res. 2017; 6: 1219, doi: 10.12688/f1000research.10903.1, indexed in Pubmed: 28781767.

3. Pasquinelli AE. MicroRNAs and their targets: recognition, regulation and an emerging reciprocal relationship. Nat Rev Genet. 2012; 13(4): 271-282, doi: 10.1038/nrg3162, indexed in Pubmed: 22411466 .

4. Santovito D, Mezzetti A, Cipollone F. MicroRNAs and atherosclerosis: new actors for an old movie. Nutr Metab Cardiovasc Dis. 2012; 22(11): 937-943, doi: 10.1016/j.numecd.2012.03.007, indexed in Pubmed: 22748605.

5. Kabłak-Ziembicka A, Przewłocki T, Stępień E, et al. Relationship between carotid intima-media thickness, cytokines, atherosclerosis extent and a two-year cardiovascular risk in patients with arteriosclerosis. Kardiol Pol. 2011; 69(10): 1024-1031, doi: 10.5603/KP.a2017.0243, indexed in Pubmed: 22006602.

6. Kehl DW, Iqbal N, Fard A, et al. Biomarkers in acute myocardial injury. Transl Res. 2012; 159(4): 252-264, doi: 10.1016/j. trsl.2011.11.002, indexed in Pubmed: 22424429.

7. Luengo-Fernandez R, Paul NLM, Gray AM, et al. Oxford Vascular Study. Population-based study of disability and institutionalization after transient ischemic attack and stroke: 10-year results of the Oxford Vascular Study. Stroke. 2013; 44(10): 2854-2861, doi: 10.1161/STROKEAHA.113.001584, indexed in Pubmed: 23920019.

8. Lovett JK, Coull AJ, Rothwell PM. Early risk of recurrence by subtype of ischemic stroke in population-based incidence 
studies. Neurology. 2004; 62(4): 569-573, doi: 10.1212/01. wnl.0000110311.09970.83, indexed in Pubmed: 14981172.

9. Urbich C, Kuehbacher A, Dimmeler S. Role of microRNAs in vascular diseases, inflammation, and angiogenesis. Cardiovasc Res. 2008; 79(4): 581-588, doi: 10.1093/cvr/cvn156, indexed in Pubmed: 18550634.

10. Ji R, Cheng Y, Yue J, et al. MicroRNA expression signature and antisense-mediated depletion reveal an essential role of MicroRNA in vascular neointimal lesion formation. Circ Res. 2007; 100(11): 1579-1588, doi: 10.1161/CIRCRESAHA.106.141986, indexed in Pubmed: 17478730.

11. Jickling GC, Ander BP, Zhan X, et al. microRNA expression in peripheral blood cells following acute ischemic stroke and their predicted gene targets. PLoS One. 2014; 9(6): e99283, doi: 10.1371/journal.pone.0099283, indexed in Pubmed: 24911610.

12. Libby P, Ridker PM, Hansson GK, et al. Leducq Transatlantic Network on Atherothrombosis. Inflammation in atherosclerosis: from pathophysiology to practice. J Am Coll Cardiol. 2009; 54(23): 2129-2138, doi: 10.1016/j.jacc.2009.09.009, indexed in Pubmed: 19942084.

13. Pieniążek P, Tekieli L, Musiałek P, et al. Carotid artery stenting according to the tailored-CAS algorithm is associated with a low complication rate at 30 days: data from the TARGET-CAS study. Kardiol Pol. 2012; 70(4): 378-386, indexed in Pubmed: 22528713.

14. Navickas R, Gal D, Laucevičius A, et al. Identifying circulating microRNAs as biomarkers of cardiovascular disease: a systematic review. Cardiovasc Res. 2016; 111(4): 322-337, doi: 10.1093/cvr/cvw174, indexed in Pubmed: 27357636.

15. Maitrias P, Metzinger-Le Meuth V, Massy ZA, et al. MicroRNA deregulation in symptomatic carotid plaque. J Vasc Surg. 2015; 62(5): 1245-1250, doi: 10.1016/j.jvs.2015.06.136, indexed in Pubmed: 26238333.

16. Cipollone F, Felicioni L, Sarzani R, et al. A unique microRNA signature associated with plaque instability in humans. Stroke. 2011; 42(9): 2556-2563, doi: 10.1161/STROKEAHA.110.597575, indexed in Pubmed: 21817153.
17. Raitoharju E, Oksala N, Lehtimäki T. MicroRNAs in the atherosclerotic plaque. Clin Chem. 2013; 59(12): 1708-1721, doi: 10.1373/clinchem.2013.204917, indexed in Pubmed: 23729638.

18. Olivieri F, Antonicelli R, Lorenzi M, et al. Diagnostic potential of circulating miR-499-5p in elderly patients with acute non ST-elevation myocardial infarction. Int J Cardiol. 2013; 167(2): 531-536, doi: 10.1016/j.ijcard.2012.01.075, indexed in Pubmed: 22330002

19. Adachi T, Nakanishi M, Otsuka Y, et al. Plasma microRNA 499 as a biomarker of acute myocardial infarction. Clin Chem. 2010; 56(7): 1183-1185, doi: 10.1373/clinchem.2010.144121, indexed in Pubmed: 20395621.

20. van Rooij E, Sutherland LB, Qi X, et al. Control of stress-dependent cardiac growth and gene expression by a microRNA. Science. 2007; 316(5824): 575-579, doi: 10.1126/science.1139089, indexed in Pubmed: 17379774.

21. Tang Y, Zheng J, Sun Y, et al. MicroRNA-1 regulates cardiomyocyte apoptosis by targeting Bcl-2. Int Heart J. 2009; 50(3): 377-387, doi: 10.1536/ihj.50.377, indexed in Pubmed: 19506341.

22. Gacoń J, Kabłak-Ziembicka A, Stępień E, et al. Decision-making microRNAs (miR-124, -133a/b, -34a and -134) in patients with occluded target vessel in acute coronary syndrome. Kardiol Pol. 2016; 74(3): 280-288, doi: 10.5603/KP.a2015.0174, indexed in Pubmed: 26365938 .

23. Dolz S, Górriz D, Tembl JI, et al. Circulating MicroRNAs as Novel Biomarkers of Stenosis Progression in Asymptomatic Carotid Stenosis. Stroke. 2017; 48(1): 10-16, doi: 10.1161/STROKEAHA.116.013650, indexed in Pubmed: 27899750.

24. Widera C, Gupta SK, Lorenzen JM, et al. Diagnostic and prognostic impact of six circulating microRNAs in acute coronary syndrome. J Mol Cell Cardiol. 2011; 51(5): 872-875, doi: 10.1016/j. yjmcc.2011.07.011, indexed in Pubmed: 21806992.

25. Gidlöf O, Smith JG, Miyazu K, et al. Circulating cardio-enriched microRNAs are associated with long-term prognosis following myocardial infarction. BMC Cardiovasc Disord. 2013; 13: 12, doi: 10.1186/1471-2261-13-12, indexed in Pubmed: 23448306.

Cite this article as: Gacoń J, Badacz R, Stępień E, et al. Diagnostic and prognostic micro-RNAs in ischaemic stroke due to carotid artery stenosis and in acute coronary syndrome: a four-year prospective study. Kardiol Pol. 2018; 76(2): 362-369, doi: 10.5603/KP.a2017.0243. 